Running title: Oxygen effects on MSC proliferation and differentiation

Continuous and uninterrupted oxygen tension influences the colony formation and oxidative metabolism of human mesenchymal stem cells

Girish Pattappa, PhD. ${ }^{1,2}$; Stephen D. Thorpe, PhD. ${ }^{1}$; Nick C. Jegard, MEng ${ }^{1}$;

Hannah K. Heywood, PhD ${ }^{1}$; Joost D. de Bruijn, PhD., ${ }^{1}$ and David A. Lee. PhD ${ }^{1 *}$

${ }^{1}$ School of Engineering and Materials Science, Queen Mary University of London, Mile End Road, London, UK. E1 4NS

${ }^{2}$ Musculoskeletal Regeneration group, AO Research Institute, Clavadelerstrasse 8. CH7270 Davos Platz, Switzerland.

*Corresponding author

Keywords

- Oxygen tension

- Mesenchymal stem cells

- Chondrogenesis

- Osteogenesis

- Metabolism

- Senescence 


\section{Author contact information}

\section{Dr. Girish Pattappa}

Musculoskeletal regeneration group,

AO Research Institute,

Clavadelerstrasse 8.

Davos Platz 7270.

Switzerland

Stephen D. Thorpe

School of Engineering and Materials Science,

Queen Mary University of London,

Mile End Road

London, E1 4NS.

UK

Nick C. Jegard

School of Engineering and Materials Science,

Queen Mary University of London,

Mile End Road

London, E1 4NS.

UK

Dr. Hannah K. Heywood

School of Engineering and Materials Science,

Queen Mary University of London,

Mile End Road

London, E1 4NS.

UK

Prof. Joost D. de Bruijn

School of Engineering and Materials Science,

Queen Mary University of London,

Mile End Road

London, E1 4NS.

UK
Tel: +41 (0)81 4142318

Fax: +41 (0)814142288

E-mail:girish.pattappa@aofoundation.org
Tel: +44 (0)207 8825194

Fax: +44 (0)207 8823390

E-mail: s.thorpe@qmul.ac.uk
Tel: +44 (0)777 1364539

Fax: +44 (0)207 8823390

E-mail:nick.jegard@gmail.com
Tel: +44 (0)207 8825368

Fax: +44 (0)207 8823390

E-mail:h.k.heywood@qmul.ac.uk
Tel: +44 (0)207 8826310

Fax: +44 (0)207 8823390

E-mail: j.d.debruijn@qmul.ac.uk 


\section{Prof. David A Lee}

School of Engineering and Materials Science,

Queen Mary University of London,

Mile End Road

Tel: +44 (0)207882 8874

London, E1 4NS.

Fax: +44 (0)207 8823390

UK

E-mail: D.A.Lee@qmul.ac.uk 


\begin{abstract}
Mesenchymal stem cells (MSCs) are an attractive cell source for tissue engineering applications due to their multi-potentiality and increased expansion potential compared to mature cells. However, the full potential of MSCs for cellular therapies is not realised, due, in part, to premature proliferative senescence and impaired differentiation capacity following expansion under $20 \%$ oxygen. Bone marrow MSCs reside under reduced oxygen levels (4-7\% oxygen), thus this study investigates the effects of uninterrupted physiological oxygen tensions $(2 \%, 5 \%)$ on MSC expansion and subsequent differentiation.
\end{abstract}

Expansion potential was evaluated from colony formation efficiency, population doubling rates and cellular senescence. Colony formation was significantly reduced under $5 \%$ oxygen compared to $2 \%$ and $20 \%$ oxygen. Population doubling time was initially shorter with $20 \%$ oxygen, but subsequently no significant differences in doubling time were detected between the oxygen conditions. MSCs expanded with $20 \%$ oxygen contained a greater proportion of senescent cells than those under physiological oxygen levels, indicated by 3-4 fold increase in $\beta$-galactosidase staining. This may be related to the $\sim 2$-fold enhanced mitochondrial oxygen consumption under this culture conditions.

Chondrogenic differentiation was achieved following expansion at each oxygen condition. However, osteogenesis was only achieved for cells expanded and differentiated at $20 \%$ oxygen, indicated by alkaline phosphatase activity and alizarin red staining. These studies demonstrate that uninterrupted hypoxia may enhance longterm MSC expansion but results in a population with impaired osteogenic differentiation potential. Thus, novel differentiation conditions are required to enable differentiation to non-chondrogenic lineages using hypoxia-cultured MSCs. 


\section{Introduction}

Bone marrow derived mesenchymal stem cells (MSCs) provide a promising source of cells for musculoskeletal tissue engineering applications due to their ability to differentiate towards various lineages including cartilage, bone, fat, tendon and muscle . In vivo, the cell resides under hypoxic oxygen tensions, ranging between 4$7 \%{ }^{1,2}$. However, the utilisation of MSCs within cellular therapies typically requires the expansion of the cells in culture to generate sufficient numbers, whilst maintaining their differentiation characteristics. Recent studies have observed that culture of MSCs under normoxic conditions (20\% oxygen) results in premature senescence and a reduction in MSC differentiation capacity with each subsequent population doubling or passage ${ }^{3,4}$. Accordingly considerable recent attention has been placed on the investigation of MSC isolation and expansion under low oxygen conditions.

Recent studies have shown that the expansion of MSCs under hypoxia or normoxia (20\% oxygen) influences MSC proliferation, senescence and differentiation 3,5,6. While greater rates of proliferation have been reported under normoxic conditions during the early stages in culture, normoxic MSCs undergo fewer total population doublings compared to hypoxic MSCs due to the induction of premature senescence ${ }^{7-}$ 10

. These effects are potentially, related to a shift in cellular energy metabolism, from a predominantly glycolytic to oxidative metabolism, with the associated release of reactive oxygen species. However detailed analysis of the metabolic profile of MSCs expanded under varying oxygen conditions remains lacking.

The initial colony forming capacity of MSC may also be influenced by cultured oxygen levels. Studies have described the isolation under hypoxia of MSC subpopulations, such as marrow isolated adult multi-lineage inducible cells (MIAMI) ${ }^{8,11}$. Furthermore, these studies have suggested that MSCs expanded under hypoxia are able to express the embryonic stem cell gene, OCT-4 and maintain their multipotency at later population doublings when differentiated under normoxia ${ }^{8,9,12,13}$. Lennon et. al. (2001) showed that MSC differentiation towards chondrogenic and osteogenic lineages was enhanced under hypoxia, although recent studies have suggested that osteogenic differentiation is inhibited under hypoxia ${ }^{13-16,16,17}$. Hypoxia enables and in some cases, enhances chondrogenic differentiation ${ }^{6,11,18}$, although Malladi et. al. 
(2006) showed that hypoxic culture inhibited chondrogenesis in micromass cultures due to the development of anoxic conditions within the micromass.

Overall the data from previous studies in relation to the effects of hypoxia on the expansion and differentiation of MSCs are inconsistent. This may be due to marked differences in the oxygen conditions used, with most studies using a single hypoxic condition, the species of the cells and their initial isolation conditions. A further limitation of previous studies is that the cells were not cultured under continuous and uninterrupted hypoxic conditions, providing a compounding variable, whereby cells under hypoxic conditions are regularly subjected to fluctuating oxygen levels, that may rise to $20 \%$ oxygen during cell manipulation, associated with passage ${ }^{5,6,9}$. Thus, the current study reports the effect of expansion of MSCs from initial bone marrow isolation under continuous and uninterrupted cultured oxygen conditions of $20 \%, 5 \%$ and $2 \%$ oxygen. The effects of the cultured oxygen conditions on initial colony formation, cell phenotype at first passage, subsequent population doubling, energy metabolism and differentiation potential were assessed. Based upon the previous literature findings, it was hypothesized that human MSCs can undergo greater population doublings without an increase in cellular senescence when cultured under hypoxia. Additionally hypoxia is hypothesised to enhance chondrogenic differentiation whilst inhibiting osteogenic differentiation. 


\section{Materials and Methods}

\section{Bone marrow culture and MSC expansion}

Bone marrow aspirates from four donors ( 3 male, 1 female) aged 21-28 were procured from a commercial source (Lonza ${ }^{\mathrm{TM}}$, Wokingham, UK). The samples were opened inside an Xvivo ${ }^{\mathrm{TM}}$ controlled oxygen culture system (Biospherix ${ }^{\mathrm{TM}}$, Redfield, New York. USA) set at 5\% oxygen. The workstation contains a microscope and incubators to enable visualisation and culture of MSCs and enables cell manipulation under appropriate oxygen conditions to ensure that the cells are maintained under continuous and uninterrupted oxygen conditions. The environment is controlled using software that monitors oxygen and carbon dioxide levels through sensors inside the workspaces and incubators. The sensitivity of the oxygen levels within the incubators and workstation is $+0.1 \%$. The $37^{0} \mathrm{C} / 5 \% \mathrm{CO}_{2}$ incubators were set at gas phase oxygen levels, 20\%, 5\% and 2\% oxygen. MSC expansion medium, $\alpha$-MEM (Invitrogen, Paisley, UK) $+8.5 \%$ FBS (Sigma-Aldrich, Poole, UK), $1 \mathrm{ng} / \mathrm{ml}$ basic fibroblast growth factor (Serotec, Oxford, UK), 100U/ml penicillin $/ 100 \mu \mathrm{g} / \mathrm{ml}$ streptomycin, 2mM L-glutamine, 25mM HEPES buffer and 0.2mM L-ascorbic acid-2-phosphate (all Sigma-Aldrich, Poole, UK), was equilibrated at each of the oxygen tensions prior to supplementation. Marrow samples were suspended in medium equilibrated at 5\% oxygen and the number of mononuclear cells was counted. The cell suspensions were seeded into $25 \mathrm{~cm}^{2}$ flasks at a density of $5 \times 10^{5}$ mononuclear cells $/ \mathrm{cm}^{2}$ and randomly allocated into equal sets of flasks to be incubated at each of the stated oxygen tensions. Each were fed equilibrated medium at their respective oxygen tension and cultured in the appropriate incubator. At the first medium exchange, six days after initial plating, culture flasks were washed with phosphate buffered saline (PBS; Sigma-Aldrich, Poole, UK) prior to addition of medium.

Throughout the initial culture period, a systematic analysis of colony formation was performed. A colony was defined as a group of 16 or more cells. Colony diameter and cell morphometric analysis was determined from representative photomicrographs using SigmaScan Pro Version 5 (SYSTAT Software Inc., CA. USA). Medium was refreshed every 2-3 days until cells within the colonies began to develop multi-layers at which point the cells were passaged by trypsinisation and the number of cells 
recovered at each oxygen condition was recorded (days 21 and 14 for donors A and B respectively). The recovered cells were seeded into culture flasks at $2 \times 10^{3}$ cells $/ \mathrm{cm}^{2}$. MSCs were allowed to proliferate under their designated oxygen conditions for a further four passages. Growth curves were derived from the data and used to calculate cell doubling time.

\section{Phenotypic characterisation of MSCs and cell cycle analysis}

The expression of positive and negative surface markers for MSCs was assessed using flow cytometry once colonies had begun to overlap; 18 days after initial plating ${ }^{19}$. Cells from two separate donors at each oxygen tension were detached from flasks using oxygen equilibrated Accutase (Sigma-Aldrich) and fixed with oxygen equilibrated $0.5 \%$ PFA for $10 \mathrm{~min}$. Cells were washed in PBS and incubated with antibodies indicated in table 1 for $30 \mathrm{~min}$ at room temperature. Following surface staining, cells were re-suspended in $0.25 \%$ triton-X100 (Sigma-Aldrich) with 2 $\mu \mathrm{g} / \mathrm{mL}$ DAPI (Invitrogen) for cell cycle analysis. Cells were analysed using 5-colour flow cytometry with an LSRII flow cytometer (BD Biosciences, Oxford, UK). Cells were gated on forward scatter and side scatter to exclude cell debris, and DAPI exclusion was used to remove non-specific fluorescence by dying/dead cells.

Furthermore, cells were taken after detachment and seeded on coverslips at $5 \times 10^{3}$ cells $/ \mathrm{cm}^{2}$. Following 24 hours incubation, coverslips were fixed with 4\% PFA for 10 min at room temperature, washed in PBS, permeabilised with $0.5 \%$ Triton-X100, blocked in goat serum and incubated overnight at $4^{\circ} \mathrm{C}$ with the primary antibody, mouse monoclonal IgM anti-STRO-1 (1:50, $200 \mu \mathrm{g} / \mathrm{mL}$, Santa Cruz Biotechnology, Heidelberg, Germany). Coverslips were washed using PBSand were incubated for 1 hour at room temperature using the secondary antibody, goat anti-mouse $\operatorname{IgG}(\mathrm{H}+\mathrm{L})$ conjugated to Alexa Fluor 488 (1:200, $2 \mathrm{mg} / \mathrm{mL}$, Invitrogen). Coverslips were counterstained with $8 \mu \mathrm{M}$ Hoechst 33342 (Invitrogen) and mounted with ProLong Gold anti-fade reagent (Invitrogen). Samples were imaged using a Leica DMI4000B inverted epifluorescent microscope fitted with a Leica DFC300 FX camera.

\section{$\beta$-galactosidase staining}


$\beta$-galactosidase staining was used as a marker of cell senescence. Cells were assessed after five passages in culture using a commercially available histochemical kit for $\beta$ galactosidase activity (Sigma-Aldrich, Poole, UK). Cells were seeded at $2 \times 10^{3}$ cells $/ \mathrm{cm}^{2}$ into 6-well plates and cultured until reaching 50-70\% confluence. The cells were fixed and stained according to manufacturers' instructions. The number of $\beta$ galactosidase-positive cells (blue stained cells) was counted in the whole area of the 6-well plate alongside the total cell number and both were recorded.

\section{Measurements of metabolite consumption and production of MSCs}

\section{(i) Measurement of oxygen consumption}

Passage 3 and 4 MSCs expanded under each oxygen condition were trypsinised at 80 - 90\% confluence and re-suspended in $\alpha$-MEM without phenol red (Invitrogen, Paisley, UK) $+8.5 \%$ FBS, pre-equilibrated at $20 \% \mathrm{O}_{2} / 5 \% \mathrm{CO}_{2}$. Cell suspensions were aliquoted into the wells of a 384-well oxygen biosensor plate (BD Bioscience, Oxford, UK) and separate 384-well plates (BD Biosciences, Oxford, UK) at a volume of $130 \mu \mathrm{l}$ with each well containing $1.5 \times 10^{5}$ cells. MSCs were additionally assessed in the presence of $10 \mathrm{mM}$ sodium azide. A zero oxygen control $(0.1 \mathrm{M}$ sodium sulphite, Sigma-Aldrich, Poole, UK) and cell-free oxygen and temperature equilibrated medium samples were also included.

The plates were sealed using a plate sealer and the biosensor plate was placed in a fluorometer (Fluostar Galaxy, BMG technologies, Aylesbury, UK) that was setup to measure fluorescence under incubator conditions at an excitation wavelength, $485 \mathrm{~nm}$, and emission wavelength, $590 \mathrm{~nm}$. The remaining plates were stored in a $37^{\circ} \mathrm{C} / 5 \%$ $\mathrm{CO}_{2}$ incubator. Oxygen consumption was measured every ten minutes for a two hour period and the rate of consumption was calculated using previously described protocols ${ }^{20-22}$. A correction factor for oxygen ingress was applied due to oxygen leakage into the well. The calculation of the consumption and per cell consumption rate have been detailed previously ${ }^{23}$. The permeability constant used in the present investigation was $0.23 \pm 0.04($ mean \pm S.D. $)$.

(ii) Measurement of glucose consumption and lactate production 
Medium was removed from 384-well plates at 1, 2, 4 and 6 hour time points for measurement of glucose consumption and lactate production within the medium using previously described assays ${ }^{21}$. The rate of glucose consumption and lactate production were derived from the linear portion of the glucose-time and lactate-time curves. These rates were normalised to cell number to obtain glucose consumption and lactate production rates.

\section{Differentiation studies: Osteogenic culture}

Passage 2 MSCs expanded under each oxygen condition were seeded at a density of 2 $\mathrm{x} 10^{3}$ cells $/ \mathrm{cm}^{2}$ into well plates (Corning, Acton, USA) and incubated under their expansion oxygen conditions. Following 24 hours after seeding, medium was replaced with oxygen-equilibrated osteogenic medium ( $\alpha$-MEM (Invitrogen, Paisley, UK) + $10 \%$ FBS $+0.05 \mathrm{mM}$ L-ascorbic acid-2-phosphate $+10 \mathrm{mM} \beta$-glycerophosphate + $0.1 \mathrm{mM}$ dexamethasone (all Sigma-Aldrich, Poole, UK)), whilst controls for the experiments were $20 \%$ oxygen MSCs fed with $\alpha$-MEM $+10 \%$ FBS. Oxygenequilibrated medium was refreshed every 2-3 days and cells were grown for 21 days.

Calcium deposition was assessed after 21 days using alizarin red staining. Alkaline phosphatase activity was measured using a commercial kit (Randox Laboratories, Country Antrim, UK), according to manufacturers' instructions. Prior to analysis, cells were lysed using 0.2\% Triton X-100 (Sigma-Aldrich, Poole, UK) in PBS. ALP concentration was normalised to the DNA concentration that was measured using the Quant-it Picogreen assay kit (Invitrogen, Paisley, UK) according to manufacturers' instructions.

A further experiment repeated the experimental setup only for cells pre-expanded at $20 \%$ and $2 \%$ oxygen. However, MSCs from each expansion oxygen condition were then differentiated at either $2 \%$ or $20 \%$ oxygen. Controls for these studies were cells differentiated at their expansion oxygen tension and cells in proliferation medium.

\section{Chondrogenic culture}


Cell pellets containing $2.5 \times 10^{5}$ cells were prepared from Passage 2 MSCs from each oxygen tension. Pellets were cultured in oxygen-equilibrated chondrogenic medium (Dulbecco's Modified Eagle Medium - High glucose (DMEM-HG) + 1mM sodium pyruvate $+0.35 \mathrm{mM}$ L-Proline $+0.17 \mathrm{mM}$ L-ascorbic acid-2-phosphate $+0.1 \mu \mathrm{M}$ dexamethasone (all Sigma-Aldrich, Poole, UK) + 10nM TGF- $\beta 3$ (Peprotech, UK) + ITS + premix (BD Biosciences, Oxford, UK)) and were incubated at their expansion oxygen tension for 21 days. Oxygen-equilibrated chondrogenic medium was fed every 2-3 days. The control for the study was pellets pre-expanded at $20 \%$ oxygen that were fed medium without TGF- $\beta 3$.

Representative pellets for each oxygen tension were removed on days $1,7,14$ and 21 for GAG and DNA analysis. Pellets were digested in the presence of papain and then DNA and total GAG content was measured using Hoescht 33258 and 1,2-dimethylene blue assay respectively ${ }^{24,25}$. Representative pellets for each oxygen condition were fixed and then embedded into paraffin blocks. Sections were created using a microtome saw ( $5 \mu \mathrm{m}$ thick) and floated on coated slides prior to staining with $1 \%$ toluidine blue solution for visualisation of GAGs within the pellet.

\section{Statistical analysis}

Colony number and cells per colony were assessed within their respective donor. Comparisons were made using single factor ANOVA $(\alpha=0.05)$. If there was significance within the data $(\mathrm{p}<0.05)$, then post-hoc student t-test with Bonferroni correction was applied. Population doubling times were assessed within their respective donor, although statistical comparison between doubling time and oxygen tension were assessed using two factor ANOVA $(\alpha=0.05)$ and then the same posthoc test was used. Data was pooled for the remaining studies and comparisons were made using single factor ANOVA. Surface marker expression was compared using a two factor ANOVA (donor and oxygen tension); Tukey's post hoc test was used for inter-group comparisons.

Colony diameter and cell area were assessed using non-parametric methods after testing the data for normality. Comparisons between groups were tested using 
Kruskal-Wallis test $(\alpha=0.05)$ and if found significant $(\mathrm{p}<0.05)$, then a MannWhitney U-test was applied. 


\section{Results}

\section{Low oxygen culture modulates colony frequency and size}

MSC colonies were first observed at each oxygen tension after six days in culture (Fig. 1a-c). The development of colonies was monitored until first trypsinisation, with data presented for the day prior to first trypsinisation (days 20 and 13 for donors A and B; Fig. 1d). No significant difference in the number of colonies was observed at $20 \%$ and $2 \%$ oxygen, whereas a significant decrease in colony number was observed for $5 \%$ oxygen cultures (Fig. 1d, Student t-test; $\mathrm{p}<0.05$ ). At first trypsinisation (days 21 and 14 for donors $\mathrm{A}$ and $\mathrm{B}$ ) the greatest number of cells were recovered for the $20 \%$ oxygen conditions (Fig. 1e), with significantly fewer cells recovered under either the $5 \%$ or $2 \%$ oxygen conditions for both donors (Student t-test; $\mathrm{p}<0.05$ ).

Moreover, morphometric analysis of colony diameter and individual cell area revealed differences for colonies formed at differing oxygen tension (Fig. 2). The diameters of colonies formed at $2 \%$ oxygen were significantly smaller than at either $20 \%$ or $5 \%$ oxygen (Fig. 2a, Mann-Whitney U-test; $\mathrm{p}<0.05$ ). Moreover, there were significant differences in individual cell area for MSCs cultured under 2\% oxygen compared with either $20 \%$ or $5 \%$ oxygen (Fig. 2 b, Mann-Whitney U-test; $p<0.05$ ).

\section{Hypoxic isolation and culture enhances expression of MSC specific surface markers}

Once colonies began to overlap after initial plating, adherent cells were seeded on coverslips and immuno-stained, or analysed using flow cytometry. MSCs were negative for CD34, CD11b, CD19, CD45 and HLA-DR, and positive to differing extents for CD90, CD44, CD105 and CD73 (Fig. 3). Isotype control plots are shown in Supplementary Figure 1. The proportion of cells highly expressing CD44, CD73 and CD105 increased with a reduction in oxygen tension for both donors (Fig. 3a). Expression of surface marker CD90 was greater at $2 \%$ oxygen for donor $\mathrm{A}$, when compared to $5 \%$ and $20 \%$ oxygen tensions (Fig. $3 \mathrm{a} ; p<0.05$ ). However there was no difference in CD90 expression for donor B. Cells positive for CD73 and CD105 were also largely positive for CD44 and CD90 (Fig. 3c). Interestingly, of the cells positive for both CD73 and CD105, a greater proportion were undergoing cell division than that of the population as a whole (Supplementary Figure 2), suggesting that the 
$\mathrm{CD} 3^{+} \mathrm{CD} 105^{+}$sub-population is highly proliferative. Culture at low oxygen tensions served to increase the proportions of cells highly expressing these markers (Figure 3b). Immunofluorescent staining for STRO-1 was most intense at $20 \%$ and $2 \%$ oxygen tensions, although there was a drop in intensity at 5\% oxygen (Supplementary Figure 3).

Hypoxia cultured MSCs show no differences in population growth rate after initial phase but have reduced cellular senescence

Assessment of MSC expansion under each oxygen condition following first trypsinisation revealed a more rapid fold increase in cell number at $20 \%$ oxygen compared with $5 \%$ or $2 \%$ oxygen, assessed over four passages (Figure $4 \mathrm{~d}$ and $4 \mathrm{e}$ ). In terms of cell doubling time (Table 2), there was a significantly shorter MSC doubling time at P1 cultured under normoxia compared with $5 \%$ or $2 \%$ oxygen for both donors (Student t-test; $\mathrm{p}<0.05$ ). However, cells expanded at $20 \%$ oxygen, significantly increased their doubling time with each subsequent passage (Student t-test; $p<0.05$ ), resulting in no significant differences in doubling time between oxygen conditions from passage 3 onwards for donor B and passage 2 onwards for donor A (ANOVA; $p$ $>0.05)$.

After five passages, cells expanded under normoxia contained a sub-population of cells that had a larger and more flattened morphology which was not evident for cells cultured at $5 \%$ and $2 \%$ oxygen, that maintained their fibroblastic morphology throughout the culture period (Fig. 5a and b). Morphometric analysis also demonstrated that the median cell area for cells cultured at $20 \%$ oxygen was significantly greater than at $5 \%$ and $2 \%$ (data not shown). A significantly greater proportion of cells cultured at $20 \%$ oxygen stained for $\beta$-galactosidase compared with either $5 \%$ or $2 \%$ oxygen, indicating greater cellular senescence (Fig. $4 \mathrm{c}$, Student t-test, $\mathrm{p}<0.05)$.

\section{Normoxia cultured MSCs have increased oxidative phosphorylation utilisation}

MSC oxygen consumption rate (Table 3) for cells expanded at 20\% oxygen was significantly higher than cells expanded at either $5 \%$ or $2 \%$ oxygen (Table 3 , Student t-test; $\mathrm{p}<0.05)$. Data were similar for both donors and were thus pooled. 
Furthermore, MSCs expanded at 20\% oxygen had a significant reduction in lactate production, compared with cells expanded at $5 \%$ or $2 \%$ oxygen (Student t-test; $\mathrm{p}<$ 0.05). There was no significant difference in consumption and production rates for MSCs expanded at either $5 \%$ or $2 \%$ oxygen (Student t-test; $\mathrm{p}>0.05$ ).

Sodium azide sensitive oxygen consumption enabled calculation of mitochondrial oxygen consumption for each expansion oxygen condition (20\% oxygen: 82.9 fmol $/ \mathrm{hr} /$ cell, $5 \%$ oxygen: $30.2 \mathrm{fmol} / \mathrm{hr} /$ cell, $2 \%$ oxygen: $40.2 \mathrm{fmol} / \mathrm{hr} /$ cell). ATP production derived from oxidative phosphorylation was approximately $35 \%$ for MSCs expanded under $20 \%$ oxygen, whilst for MSCs expanded under either $5 \%$ or $2 \%$ oxygen, a significantly reduced ATP generation occurred via this pathway $(\sim 15 \%)$.

\section{Hypoxia cultured MSCs have reduced capacity for osteogenesis under normoxia or hypoxia}

Figure 6e demonstrates an increase in GAG content with time in culture for each oxygen tension. There were no significant differences in the GAG/DNA ratio under chondrogenic stimulation either between oxygen tensions and time points (ANOVA; $\mathrm{p}>0.05)$. However, the GAG/DNA ratio for the chondrogenic pellets was significantly greater than unstimulated pellets (Student t-test; $\mathrm{p}<0.05$ ). This was further demonstrated through toluidine blue staining (Fig. 5a-d).

MSCs expanded and differentiated at 20\% oxygen exhibited enhanced alkaline phosphatase activity compared to $5 \%$ and $2 \%$ oxygen cultures (Fig. 7 a). There were no significant differences in alkaline phosphatase activity between samples expanded and differentiated under hypoxia and control cultures (ANOVA; $p>0.05$ ). This was further demonstrated via alizarin red staining for calcium deposition (Fig. 7b-e).

MSCs expanded at $20 \%$ and differentiated at $2 \%$ oxygen demonstrated limited alkaline phosphatase activity and no alizarin red staining compared to cells differentiated at $20 \%$ oxygen (Fig. 8a-c, Student t-test, $\mathrm{p}<0.05$ ). None of the cells expanded at $2 \%$ oxygen expressed appreciable alkaline phosphatase activity (Fig. 8a) or alizarin red staining (Fig. $8 \mathrm{~d}$ and e) under any of the differentiation conditions assessed. 


\section{Discussion}

The present study investigated the effect of hypoxia (5\% oxygen and $2 \%$ oxygen) on the isolation, proliferation and differentiation of human bone marrow derived MSCs. Regenerative medicine applications require high cell numbers for therapeutic uses. However, MSCs have demonstrated premature senescence and impaired multipotency at later population doublings upon expansion under normoxia ${ }^{3,7}$. The multipotentiality of MSCs and other stem cells, enables them to be utilised for many therapeutic applications. Thus, methods that enable maintenance of multipotency at later population doublings are required. In this instance, recent investigations have used hypoxic culture to help enhance MSC population doublings ${ }^{8-10,25}$. Furthermore, they demonstrated that at later population doublings, there was a preservation of their multipotency when conducted under normoxia. However, in the present study, continuous and uninterrupted oxygen control was used from initial plating. The current data demonstrated marked differences between the three oxygen conditions from the initial colony forming stage onwards that may affect subsequent proliferation kinetics, metabolic phenotype and ability to undergo osteogenesis and chondrogenesis. Bone marrow aspirates from two donors were used and although there were quantitative differences, for example in colony formation and proliferation rates, the broad effect of hypoxia was similar between donors.

The present study showed that fewer colonies were formed from the initial adherent cell fraction within the bone marrow at $5 \%$ oxygen compared with the $20 \%$ and $2 \%$ oxygen conditions (Fig. 1d, e). These findings differ from previous studies that have demonstrated similar colony formation or enhanced colony formation under hypoxia $5,7,8$. However, methodologies adopted in some of these studies differ from the current investigation, with colony forming efficiency assessed following an initial culture period of one to four passages ${ }^{5,9}$. Fehrer et. al., 2007 did not demonstrate differences in colony formation from primary isolation between $20 \%$ and $3 \%$ oxygen, similar to the current data for $2 \%$ and $20 \%$ oxygen. D'Ippolito et. al., 2004 demonstrated robust colony formation at $3 \%$ oxygen, with colony numbers per $10^{6}$ plated mononuclear cells comparable with the current study, although they did not provide comparative data with isolation at $20 \%$ oxygen. 
Analysis of the size of the MSC colonies and individual cells therein, revealed further differences, most notably the smaller size of the colonies containing larger cells for MSCs cultured at $2 \%$ oxygen compared to the other conditions. The combined effects of colony number, colony size and cell size are consistent with the markedly reduced numbers of cells recovered at first trypsinisation for the hypoxic conditions compared to normoxia (Fig. 1e). These data are consistent with very early differences in the isolation of adherent MSCs from bone marrow at each of the oxygen conditions tested, which may reflect the selection of sub-populations of cells with differing morphologies and phenotypic characteristics.

The presence of sub-populations was reflected in the flow-cytometry analysis of MSCs from these colonies (figure 3). There was greater expression of typical MSC markers at $5 \%$ and $2 \%$ oxygen compared to normoxia. These data concur with previous literature studies, suggesting the MSC phenotype may be enhanced under low oxygen ${ }^{7,27-29}$. Furthermore, analysis of a specific MSC sub-population (CD73 and CD105 positive cells) isolated under $2 \%$ oxygen, revealed a highly proliferative cell population under these conditions (Supplementary Figure 2). It has been suggested that CD105 positive MSCs are a highly chondrogenic MSC sub-population ${ }^{30,31}$. Thus, these data indicate that typical MSC markers are highly expressed under low oxygen and that there may be specific MSC sub-populations isolated under hypoxia.

Growth kinetics were assessed from first passage. Initially, there was a clear relationship between proliferation rate and oxygen levels, with cells under hypoxia exhibiting increased population doubling times (Fig. 4d, e). However, while doubling times remained relatively constant under hypoxia, there was a trend toward increasing doubling times at later passage for cells maintained at 20\% oxygen (Table 1). These findings are consistent with previous studies that have demonstrated reduced proliferation rates under hypoxia at early passages ${ }^{5}$, although other studies have reported enhanced proliferation under hypoxia ${ }^{7,9}$. The increased doubling time with passage at $20 \%$ oxygen suggests that the cells may have approached their population doubling limit after five passages (approximately 9 population doublings from first trypsinisation). Indeed, Moussavi-Harami et. al. (2004) showed that human MSCs transfected with human telomerase reverse transcriptase (hTERT), had a population doubling limit of 11 population doublings. However, previous investigations have 
shown that MSCs cultured under hypoxia, proliferate for a longer period compared with normoxia ${ }^{5}$. The growth curves generated from the present investigation are in agreement with these previous studies. Furthermore, a greater proportion of cells expanded under $20 \%$ oxygen stained positive for the senescence marker $\beta$ galactosidase compared with hypoxic cultures (Fig. 5c), further supporting the suggestion that the cells may be reaching their population doubling limit by passage 5 and exhibiting replicative senescence. However, in order to substantiate this point, a longer culture period would be required to investigate their actual population doubling limit under each oxygen condition.

The data from the initial colony forming stage and subsequent expansion phase suggests that culture at $20 \%$ oxygen will produce greater cell numbers over a shorter period of time compared with hypoxia. Indeed, the current data suggest that over a 50 day period from initial isolation culture at $20 \%$ oxygen could generate 5 -fold increase in cumulative cells numbers compared to $2 \%$ hypoxic culture, which would therefore require approximately a further 20 days culture to generate similar numbers of cells. This has clear implications for the incorporation of hypoxic expansion for cell therapies. However, the more rapid initial proliferation must be balanced against the reduced levels of senescence observed after five passages or approximately 9 population doublings of hypoxic culture. Premature senescence under normoxia may be associated with the underlying cellular metabolism. The present investigation showed that cells expanded at $20 \%$ oxygen, had a significantly greater per-cell oxygen consumption rate and utilisation of oxidative phosphorylation compared with hypoxic cultures (Table 3). These factors may contribute to the increased cellular senescence through the production of reactive oxygen species, with associated oxidative damage 3,32

. The greater proportion of ATP production derived from glycolysis for MSCs expanded under hypoxia correlates with previous studies 5,6,33,34. Accordingly, cells expanded under hypoxia may retain a more physiological glycolytic phenotype.

MSCs are particularly attractive for tissue engineering applications due to their ability to differentiate towards various mature phenotypes. The present study showed no significant difference in the amount of GAG produced by MSCs expanded and differentiated under each of the tested oxygen conditions (Fig. 6e), which is in 
agreement with a recent study that showed no enhancement in chondrogenic gene expression under hypoxia ${ }^{37}$. However a number of other studies have suggested that hypoxia enhances MSC chondrogenesis and the re-expression of chondrocytic phenotype by dedifferentiated chondrocytes, assessed through the expression of the early chondrogenic marker, SOX-9 and the accumulation of GAG and collagen ${ }^{6,11,18}$. However, it should be noted that these studies used hypoxia during the differentiation phase only. Furthermore, recent studies have described the use of hypoxia to prevent chondrogenic hypertrophy, as hypoxic cultures had reduced collagen type $\mathrm{X}$ expression (known marker of hypertrophy) compared to normoxic cultures ${ }^{27,49}$.

In contrast, MSC osteogenesis was only displayed for MSCs expanded and differentiated under normoxia, as hypoxic cultures did not show either calcium deposition or elevated alkaline phosphatase activity (Fig. 7a, d, e). Previous investigations have demonstrated that MSCs expanded under hypoxia, were able to differentiate towards the osteogenic lineage upon culture at $20 \%$ oxygen $8,9,11,12,49$. However, the present investigation showed that cells expanded at $2 \%$ oxygen, did not express indicators of osteogenesis upon differentiation at 20\% oxygen (Fig. 8). A reason for the contrasting MSC differentiation findings during culture under hypoxia may be associated with MSC oxidative capacity and regulation of hypoxia inducible factor-1 $\alpha(\mathrm{HIF}-1 \alpha)$. The present investigation has demonstrated that MSC oxidative capacity or oxygen consumption is significantly reduced for hypoxia-expanded MSCs (Table 3). MSC osteogenesis is associated with a higher level of oxygen consumption compared with chondrogenesis and therefore reduced oxygen consumption during the differentiation process may inhibited osteogenesis ${ }^{36}$. Chen et. al. (2008) demonstrated that mitochondrial content and activity increases during osteogenesis, with an associated increase in oxygen consumption. However, inhibition of oxygen consumption using a mitochondrial inhibitor resulted in inhibited osteogenesis. Therefore, the diminished osteogenic differentiation from hypoxia-expanded MSCs may be related to the reduced oxygen consumption. Furthermore, the non-degradation of HIF-1 $\alpha$ expression that controls cell metabolism, in particular, stimulating glycolysis and its associated enzymes ${ }^{37-39}$ has been shown to inhibit osteogenicspecific genes related to MSC metabolism during osteogenesis 15,16,40,41. In the present investigation, we assessed the presence of HIF-1 $\alpha$ under each oxygen 
condition during the proliferation stages using immunofluorescence. However, we did not observe marked changes in staining intensities between each oxygen condition (data not shown).

To generate an osteogenic phenotype, an alternative differentiation route may need to be used for the process. Jukes et. al. (2008) and Farrell et. al. (2009) have demonstrated that MSCs and embryonic stem cells conditioned towards the chondrogenic lineage are able to undergo osteogenic differentiation following culture using osteogenic supplements. The process replicates endochondral ossification during bone tissue development and may be utilised for the MSCs derived from hypoxic culture within the present investigation.

The differentiation results indicate that continuous and uninterrupted hypoxia may have facilitated the selection of an MSC sub-population during earliest colonyforming stages. This was supported by increased MSC surface marker expression with reduced oxygen tension, and morphometric analysis of cells within colonies indicating the prevalence of different cellular morphologies at each oxygen tension which may impact on the differentiation capacity of the cells ${ }^{44-47}$. The present investigation suggests that chondroprogenitors were selected, correlating with results using adipose-derived MSCs under similar conditions, although further analysis for this particular hypothesis is required ${ }^{48}$. Future investigations may wish to use clonal expansion techniques to investigate differentiation potential of MSCs from different colonies under each oxygen condition and if specific early chondrogenic and osteogenic marker genes (SOX9 and Runx2) have variable expression by cells isolated under these conditions that predispose them towards a specific phenotype 4 . However, the results from the present investigation indicate that expansion under hypoxia may act to inhibit osteogenesis through reduced utilisation of oxidative phosphorylation, via hypoxia-induced modulation of their oxidative phosphorylation or mitochondrial capacity. For clinical application, hypoxic culture of MSCs during colony formation and expansion is beneficial as it delivers a greater expansion potential, but new techniques may be required to enable differentiation towards multiple lineages. 

References

1. Grant, J. L. and Smith, B. Bone marrow gas tensions, bone marrow blood flow, and erythropoiesis in man. Annals of Internal Medicine, 1963, 58: 801-809.

2. Kofoed, H., Sjontoft, E., Siemssen, S. O., and Olesen, H. P. Bone marrow circulation after osteotomy. Blood flow, $\mathrm{pO2}, \mathrm{pCO}$, and pressure studied in dogs. Acta Orthopaedica Scandinavica, 1985, 56: 400-403.

3. Moussavi-Harami, F., Duwayri, Y., Martin, J. A., Moussavi-Harami, F., and Buckwalter, J. A. Oxygen effects on senescence in chondrocytes and mesenchymal stem cells: consequences for tissue engineering. Iowa Orthopaedic Journal, 2004, 24: 15-20.

4. Muraglia, A., Cancedda, R., and Quarto, R. Clonal mesenchymal progenitors from human bone marrow differentiate in vitro according to a hierarchical model. Journal of Cell Science, 2000, 113 ( Pt 7): 1161-1166.

5. Grayson, W. L., Zhao, F., Izadpanah, R., Bunnell, B., and Ma, T. Effects of hypoxia on human mesenchymal stem cell expansion and plasticity in 3D constructs. Journal of cellular physiology, 2006, 207: 331-339.

6. Wang, D. W., Fermor, B., Gimble, J. M., Awad, H. A., and Guilak, F. Influence of oxygen on the proliferation and metabolism of adipose 
derived adult stem cells. Journal of cellular physiology, 2005, 204: 184191.

7. Dos Santos, F., Andrade, P. Z., Boura, J. S. et al. Ex vivo expansion of human mesenchymal stem cells: a more effective cell proliferation kinetics and metabolism under hypoxia. J. Cell Physiol, 2010, 223: 27-35.

8. Fehrer, C., Brunauer, R., Laschober, G. et al. Reduced oxygen tension attenuates differentiation capacity of human mesenchymal stem cells and prolongs their lifespan. Aging Cell, 2007, 6: 745-757.

9. Grayson, W. L., Zhao, F., Bunnell, B., and Ma, T. Hypoxia enhances proliferation and tissue formation of human mesenchymal stem cells. Biochem. Biophys. Res. Commun., 2007, 358: 948-953.

10. Sethe, S., Scutt, A., and Stolzing, A. Aging of mesenchymal stem cells. Ageing Res. Rev., 2006, 5: 91-116.

11. Lennon, D. P., Edmison, J. M., and Caplan, A. I. Cultivation of rat marrow-derived mesenchymal stem cells in reduced oxygen tension: effects on in vitro and in vivo osteochondrogenesis. Journal of cellular physiology, 2001, 187: 345-355.

12. d'Ippolito, G., Diabira, S., Howard, G. A. et al. Marrow-isolated adult multilineage inducible (MIAMI) cells, a unique population of postnatal 
young and old human cells with extensive expansion and differentiation potential. Journal of Cell Science, 2004, 117: 2971-2981.

13. d'Ippolito, G., Diabira, S., Howard, G. A., Roos, B. A., and Schiller, P. C. Low oxygen tension inhibits osteogenic differentiation and enhances stemness of human MIAMI cells. Bone, 2006, 39: 513-522.

14. Malladi, P., Xu, Y., Chiou, M., Giaccia, A. J., and Longaker, M. T. Effect of reduced oxygen tension on chondrogenesis and osteogenesis in adiposederived mesenchymal cells. Am. J. Physiol Cell Physiol, 2006, 290: C1139C1146.

15. Potier, E., Ferreira, E., Andriamanalijaona, R. et al. Hypoxia affects mesenchymal stromal cell osteogenic differentiation and angiogenic factor expression. Bone, 2007, 40: 1078-1087.

16. Salim, A., Nacamuli, R. P., Morgan, E. F., Giaccia, A. J., and Longaker, M. T. Transient changes in oxygen tension inhibit osteogenic differentiation and Runx2 expression in osteoblasts. Journal of Biological Chemistry, 2004, 279: 40007-40016.

17. Utting, J. C., Robins, S. P., Brandao-Burch, A. et al. Hypoxia inhibits the growth, differentiation and bone-forming capacity of rat osteoblasts. Exp. Cell Res., 2006, 312: 1693-1702. 
18. Robins, J. C., Akeno, N., Mukherjee, A. et al. Hypoxia induces chondrocyte-specific gene expression in mesenchymal cells in association with transcriptional activation of Sox9. Bone, 2005, 37: 313-322.

19. Dominici, M., Le, B. K., Mueller, I. et al. Minimal criteria for defining multipotent mesenchymal stromal cells. The International Society for Cellular Therapy position statement. Cytotherapy., 2006, 8: 315-317.

20. Dike, L. E., Xia, H., Guarino, R. D., Presnell, S. C., and Timmins, M. R. Rapid method for assessing oxygen consumption of cells from transientstate measurements of pericellular dissolved oxygen concentration. Cytotechnology, 2006.

21. Heywood, H. K., Bader, D. L., and Lee, D. A. Rate of oxygen consumption by isolated articular chondrocytes is sensitive to medium glucose concentration. Journal of cellular physiology, 2006, 206: 402-410.

22. Heywood, H. K., Knight, M. M., and Lee, D. A. Both superficial and deep zone articular chondrocyte subpopulations exhibit the crabtree effect but have different basal oxygen consumption rates. J. Cell Physiol, 2010.

23. Heywood, H. K., Knight, M. M., and Lee, D. A. Both superficial and deep zone articular chondrocyte subpopulations exhibit the crabtree effect but have different basal oxygen consumption rates. J. Cell Physiol, 2010. 
24. McGowan, K. B., Kurtis, M. S., Lottman, L. M., Watson, D., and Sah, R. L. Biochemical quantification of DNA in human articular and septal cartilage using PicoGreen and Hoechst 33258. Osteoarthritis Cartilage, 2002, 10: 580-587.

25. Farndale, R. W., Buttle, D. J., and Barrett, A. J. Improved quantitation and discrimination of sulphated glycosaminoglycans by use of dimethylmethylene blue. Biochimica et Biophysica Acta, 1986, 883: 173177.

26. DiGirolamo, C. M., Stokes, D., Colter, D. et al. Propagation and senescence of human marrow stromal cells in culture: a simple colonyforming assay identifies samples with the greatest potential to propagate and differentiate. Br. J. Haematol., 1999, 107: 275-281.

27. Adesida, A. B., Mulet-Sierra, A., and Jomha, N. M. Hypoxia mediated isolation and expansion enhances the chondrogenic capacity of bone marrow mesenchymal stromal cells. Stem Cell Res. Ther., 2012, 3: 9.

28. Basciano, L., Nemos, C., Foliguet, B. et al. Long term culture of mesenchymal stem cells in hypoxia promotes a genetic program maintaining their undifferentiated and multipotent status. BMC. Cell Biol., 2011, 12: 12. 
29. d'Ippolito, G., Howard, G. A., Roos, B. A., and Schiller, P. C. Isolation and characterization of marrow-isolated adult multilineage inducible (MIAMI) cells. Exp. Hematol., 2006, 34: 1608-1610.

30. Arufe, M. C., De la, F. A., Fuentes-Boquete, I., de Toro, F. J., and Blanco, F. J. Differentiation of synovial CD-105(+) human mesenchymal stem cells into chondrocyte-like cells through spheroid formation. J. Cell Biochem., 2009, 108: 145-155.

31. Jiang, T., Liu, W., Lv, X. et al. Potent in vitro chondrogenesis of CD105 enriched human adipose-derived stem cells. Biomaterials, 2010, 31: 35643571.

32. Martin, J. A., Klingelhutz, A. J., Moussavi-Harami, F., and Buckwalter, J. A. Effects of oxidative damage and telomerase activity on human articular cartilage chondrocyte senescence. Journals of Gerontology A Biol. Sci. Med. Sci., 2004, 59: 324-337.

33. Follmar, K. E., Decroos, F. C., Prichard, H. L. et al. Effects of glutamine, glucose, and oxygen concentration on the metabolism and proliferation of rabbit adipose-derived stem cells. Tissue Eng, 2006, 12: 3525-3533.

34. Mischen, B. T., Follmar, K. E., Moyer, K. E. et al. Metabolic and functional characterization of human adipose-derived stem cells in tissue 
engineering. Plast. Reconstr. Surg., 2008, 122: 725-738.

35. Pilgaard, L., Lund, P., Duroux, M. et al. Effect of oxygen concentration, culture format and donor variability on in vitro chondrogenesis of human adipose tissue-derived stem cells. Regen. Med., 2009, 4: 539-548.

36. Pattappa, G., Heywood, H. K., de Bruijn, J. D., and Lee, D. A. The metabolism of human mesenchymal stem cells during proliferation and differentiation. J. Cell Physiol, 2010.

37. Rajpurohit, R., Koch, C. J., Tao, Z., Teixeira, C. M., and Shapiro, I. M. Adaptation of chondrocytes to low oxygen tension: relationship between hypoxia and cellular metabolism. Journal of cellular physiology, 1996, 168: 424-432.

38. Seagroves, T. N., Ryan, H. E., Lu, H. et al. Transcription factor HIF-1 is a necessary mediator of the pasteur effect in mammalian cells. Mol. Cell Biol., 2001, 21: 3436-3444.

39. Semenza, G. L. Expression of hypoxia-inducible factor 1: mechanisms and consequences. Biochem. Pharmacol., 2000, 59: 47-53.

40. Park, J. H., Park, B. H., Kim, H. K., Park, T. S., and Baek, H. S. Hypoxia decreases Runx2/Cbfa1 expression in human osteoblast-like cells. 
Molecular and Cellular Endocrinology, 2002, 192: 197-203.

41. Warren, S. M., Steinbrech, D. S., Mehrara, B. J. et al. Hypoxia regulates osteoblast gene expression. Journal of Surgical Research, 2001, 99: 147155.

42. Farrell, E., van der Jagt, O. P., Koevoet, W. et al. Chondrogenic priming of human bone marrow stromal cells: a better route to bone repair? Tissue Eng Part C. Methods, 2009, 15: 285-295.

43. Jukes, J. M., Both, S. K., Leusink, A. et al. Endochondral bone tissue engineering using embryonic stem cells. Proc. Natl. Acad. Sci. U. S. A, 2008, 105: 6840-6845.

44. Colter, D. C., Class, R., DiGirolamo, C. M., and Prockop, D. J. Rapid expansion of recycling stem cells in cultures of plastic-adherent cells from human bone marrow. Proceedings of National of Academy of Science U. S. A, 2000, 97: 3213-3218.

45. Colter, D. C., Sekiya, I., and Prockop, D. J. Identification of a subpopulation of rapidly self-renewing and multipotential adult stem cells in colonies of human marrow stromal cells. Proceedings of National of Academy of Science U. S. A, 2001, 98: 7841-7845. 
46. Heywood, H. K. and Lee, D. A. Monolayer expansion induces an oxidative metabolism and ROS in chondrocytes. Biochem. Biophys. Res. Commun., 2008, 373: 224-229.

47. Sekiya, I., Larson, B. L., Smith, J. R. et al. Expansion of human adult stem cells from bone marrow stroma: conditions that maximize the yields of early progenitors and evaluate their quality. Stem Cells, 2002, 20: 530541.

48. Xu, Y., Malladi, P., Chiou, M. et al. In vitro expansion of adipose-derived adult stromal cells in hypoxia enhances early chondrogenesis. Tissue Eng, 2007, 13: 2981-2993.

49. Sheehy EJ, Buckley CT, Kelly DJ. Oxygen tension regulates the osteogenic, chondrogenic and endochondral phenotype of bone marrow derived mesenchymal stem cells. Biochem Biophys Res Commun. 2012 Jan 6;417(1):305-10

50. Chen CT, Shih YR, Kuo TK, Lee OK, Wei YH. Coordinated changes of mitochondrial biogenesis and antioxidant enzymes during osteogenic differentiation of human mesenchymal stem cells. Stem Cells. 2008 Apr;26(4):960-8. 
Table 1. List of antibodies used in multicolour flow cytometry. All antibodies are anti-human produced in mouse from BD Biosciences, Oxford, UK.

Table 2. The cell doubling times for cells grown under $20 \%, 5 \%$ and $2 \%$ oxygen conditions for the donors $\mathrm{A}$ and $\mathrm{B}$.

Table 3. Oxygen and glucose consumption and lactate production rate of MSCs expanded at $20 \%, 5 \%$ and $2 \%$ oxygen. Data represent mean \pm S.D. of $n=6$.

Figure 1. Representative photomicrographs of colonies formed under (a) $20 \%$, (b) $5 \%$ and (c) $2 \%$ oxygen conditions on day 8 of culture. The number of colonies per flask (d) and number of cells recovered per flask at first trypsinisation (e) at 20\%, 5\% and $2 \%$ oxygen for donor $A(\square)$ and B $(\square)$, cultures. Data represent mean \pm S.D., donor $\mathrm{A}[\mathrm{n}=4]$ and donor $\mathrm{B}[\mathrm{n}=3]$; Bonferroni corrected student t-test: $* \mathrm{p}<0.05$.

Figure 2. The (a) colony diameter and (b) individual cell area within colonies formed under $20 \%, 5 \%$ and $2 \%$ oxygen conditions. Data represent the median \pm I.Q.R.; Mann Whitney U-test: ${ }^{*} \mathrm{p}<0.05$.

Figure 3. Phenotypic characterisation of MSCs after initial plating. (a) Expression of MSC markers CD44, CD73, CD90 and CD105 as a percentage of total population at P0. (b) Percentage of CD $44^{+} \mathrm{CD} 90^{+}$cells (top) and $\mathrm{CD} 73^{+} \mathrm{CD} 105^{+}$cells (bottom). (c) Representative dot plots from Donor A for MSC markers; CD73 vs. CD105 (top) and CD44 vs. CD90 (bottom). $\mathrm{CD}^{+} 3^{+} \mathrm{CD} 105^{+}$cells are shown in grey. Data presented as mean \pm standard deviation. $*$ : $p<0.05$ vs. $20 \% \mathrm{O}_{2}$ and $5 \% \mathrm{O}_{2}, * *: p<0.01$ vs. $20 \% \mathrm{O}_{2}$ and $5 \% \mathrm{O}_{2}$, !!: $p<0.01$ vs. $20 \% \mathrm{O}_{2}$, !!!: $p<0.001$ vs. $20 \% \mathrm{O}_{2}$. As only one population of cells was analysed per donor for CD44, error bars are not included on this chart.

Figure 4. Representative photomicrographs of MSCs cultured at P1 for 5 days for cells cultured at (a) $20 \%$, (b) $5 \%$ and (c) $2 \%$ oxygen. Population growth curve in terms of potential number of MSCs during time in culture post P0 under varying oxygen conditions for (d) donor A $[20 \%(\diamond) 5 \%(-\square)$ ), 2\% (-)], and (e) donor B $[20 \%(-), 5 \%(-)$ and $2 \%(\star)]$. 
Figure 5. Representative photomicrographs of unstained $2 \%$ oxygen (a) and stained $20 \%$ oxygen (b) cultures at passage 5 . (c) The percentage of $\beta$-galactosidase positive cells at passage 5 cultured under $20 \%, 5 \%$ and $2 \%$ oxygen conditions. Data represent mean \pm S.D. of $n=6$; Donor A $(\square)$ and Donor B $(\square)$; Bonferroni corrected student ttest: ${ }^{*} \mathrm{p}<0.05$.

Figure 6. Representative photomicrographs of chondrogenic pellets stained for GAG deposition using toluidine blue for cells cultured under (a) $20 \%\left(-\mathrm{TGF} \beta_{3}\right)$, (b) $20 \%\left(+\mathrm{TGF}_{3}\right)$, (c) $5 \%$ and (d) $2 \%$ oxygen conditions after 21 days in culture. (e) A graph showing the change in GAG/DNA in chondrogenic pellets cultured under

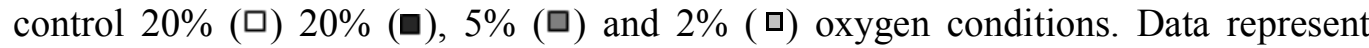
mean \pm S.D. of $\mathrm{n}=6$; Bonferroni corrected student t-test: $* \mathrm{p}<0.05$.

Figure 7. (a) Alkaline phosphatase activity of human MSCs pre-cultured and differentiated in ostoeogenic medium under $20 \%, 5 \%$ or $2 \%$ oxygen conditions. Data represent mean \pm S.D. of $n=6 ; 20 \%$ control $(\bullet), 20 \%$ osteogenesis $(-\rightarrow), 5 \%$ osteogenesis ( $\square-)$ and $2 \%$ osteogenesis ( $\bullet$ ). Representative photomicrographs of osteogenic cultures stained for calcium deposition using alizarin red for cells cultured under (b) $20 \%$ control, (c) $20 \%$, (d) $5 \%$ and (e) $2 \%$ oxygen conditions.

Figure 8. (a) Alkaline phosphatase enzyme activity for human MSCs pre-cultured under $20 \%$ and $2 \%$ oxygen and differentiated in osteogenic medium under either $20 \%$ or $2 \%$ oxygen. Data represent mean \pm SEM of $n=6 ; 20 \%-20 \%$ oxygen control $(\curvearrowright)$

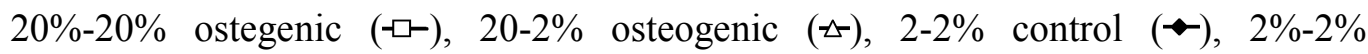
osteogenic (-) and 2-20\% osteogenic( $(₫)$. Representative photomicrographs of osteogenic cultures stained for calcium deposition for cells pre-cultured under $20 \%$ (b, c) or $2 \%$ oxygen $(\mathrm{d}, \mathrm{e})$ and differentiated under either $20 \%$ oxygen $(\mathrm{b}, \mathrm{d})$ or $2 \%$ oxygen (c, e).

Supplementary Figure 1. Representative dot plots from Donor A for isotype controls including mIgG1 $\kappa$ APC, mIgG1 $\kappa$ PerCP-Cy5.5, mIgG1 $\kappa$ FITC, mIgG1 $\kappa$ PE and mIgG2a $\kappa$ PE. 
Supplementary Figure 2. Representative imunofluorescent staining of MSCs from Donor A for STRO-1. Scale bar:

Supplementary Figure 3. Cell cycle analysis of DAPI stained cells with flow cytometry. (a) Fraction of $\mathrm{CD}^{+} 3^{+} \mathrm{CD} 105^{+}$cell sub-population in $\mathrm{S}, \mathrm{G}_{2}$ and $\mathrm{M}$ phases normalised to fraction of total analysed cells in $\mathrm{S}, \mathrm{G}_{2}$ and $\mathrm{M}$ phases. (b) Example cell cycle analysis for Donor A at $2 \%$ oxygen with gating on specific sub-populations. 


\begin{tabular}{|c|c|c|}
\hline Application & Antibody & Conjugate \\
\hline Positive marker & CD90 (Clone: 5E10) & FITC \\
\hline Positive marker & CD105 (Clone: 266) & PerCP-Сy5.5 \\
\hline Positive marker & CD73 (Clone: AD2) & APC \\
\hline Positive marker & CD44 (Clone: G44-26) & $\mathrm{PE}$ \\
\hline Negative marker & CD34 (Clone: 581) & $\mathrm{PE}$ \\
\hline Negative marker & CD11b (Clone: ICRF44) & $\mathrm{PE}$ \\
\hline Negative marker & CD19 (Clone: HIB19) & PE \\
\hline Negative marker & CD45 (Clone: HI30) & $\mathrm{PE}$ \\
\hline Negative marker & HLA-DR (Clone: G46-6) & $\mathrm{PE}$ \\
\hline CD90 Isotype control & mIgG1, к (Clone: X40) & FITC \\
\hline CD105 Isotype control & mIgG1, к (Clone: X40) & PerCP-Cy5.5 \\
\hline CD73 Isotype control & mIgG1, к (Clone: X40) & APC \\
\hline CD44 Isotype control & mIgG2b, к (Clone: 27-35) & $\mathrm{PE}$ \\
\hline Negative marker isotype control & mIgG1, к (Clone: X40) & $\mathrm{PE}$ \\
\hline Negative marker isotype control & mIgG2a, к (Clone: G155-178) & $\mathrm{PE}$ \\
\hline
\end{tabular}

Table 1 
Table 2

\begin{tabular}{|c|c|c|c|c|c|c|}
\hline \multirow{3}{*}{ Passage } & \multicolumn{6}{|c|}{ Population doubling time (hours) } \\
\hline & \multicolumn{2}{|c|}{$20 \%$} & \multicolumn{2}{|c|}{$5 \%$} & \multicolumn{2}{|c|}{$2 \%$} \\
\hline & A & B & A & B & A & B \\
\hline P1 & $99.1 \pm 20.3$ & $72.3 \pm 8.3$ & $143.9 \pm 13.2$ & $99.3 \pm 12.9$ & $164.4 \pm 21.7$ & $169.2 \pm 20.5$ \\
\hline P2 & $137.5 \pm 25.8$ & $80.9 \pm 5.1$ & $134.6 \pm 29.4$ & $146 \pm 10.9$ & $156.4 \pm 22.5$ & $184.2 \pm 34.5$ \\
\hline P3 & $139.6 \pm 7.5$ & $154.4 \pm 30.9$ & $146.9 \pm 6.9$ & $159.9 \pm 19.8$ & $165.4 \pm 37.8$ & $133.8 \pm 13.1$ \\
\hline P4 & $161.6 \pm 14.6$ & $190.0 \pm 33.0$ & $143.8 \pm 7.7$ & $147.8 \pm 24.4$ & $134.3 \pm 7.3$ & $143.6 \pm 6.6$ \\
\hline
\end{tabular}


Table 3

\begin{tabular}{|c|c|c|c|}
\hline $\begin{array}{c}\text { Expansion oxygen } \\
\text { tension }\end{array}$ & $\begin{array}{c}\text { Oxygen consumption } \\
\text { rate/cell } \\
(\mathbf{f m o l} / \mathbf{h r} / \text { cell) }\end{array}$ & $\begin{array}{c}\text { Glucose consumption } \\
\text { rate/cell (fmol/hr/cell) }\end{array}$ & $\begin{array}{c}\text { Lactate production } \\
\text { rate/cell } \\
\text { (fmol/hr/cell) }\end{array}$ \\
\hline $\mathbf{2 0 \%}$ & $119.2 \pm 18.4$ & $225.4 \pm 41.4$ & $649.5 \pm 109$ \\
\hline $\mathbf{5 \%}$ & $56.6 \pm 14.9$ & $262.4 \pm 53.3$ & $783.7 \pm 173$ \\
\hline $\mathbf{2 \%}$ & $77.0 \pm 13.9$ & $328.8 \pm 47 . .4$ & $874.4 \pm 132.8$ \\
\hline
\end{tabular}



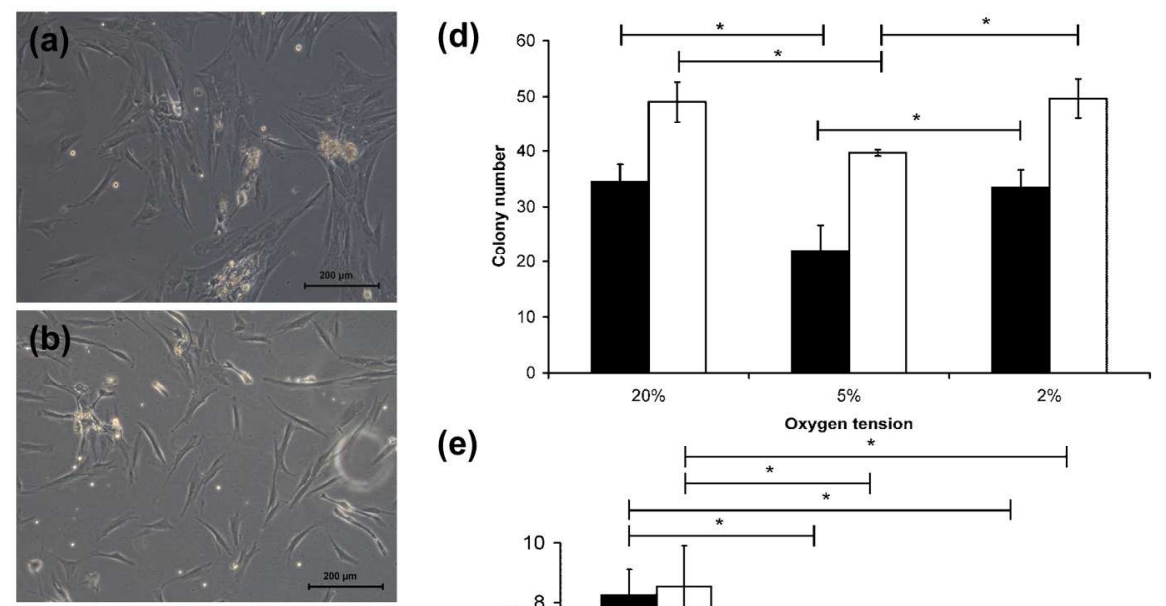

(e)

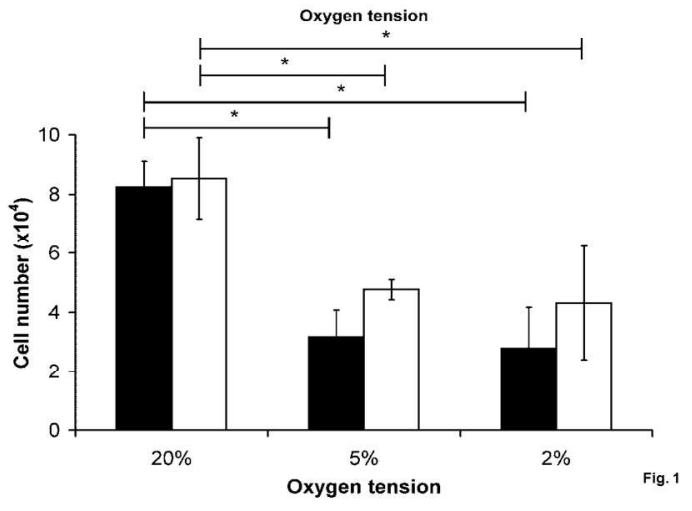


(a)

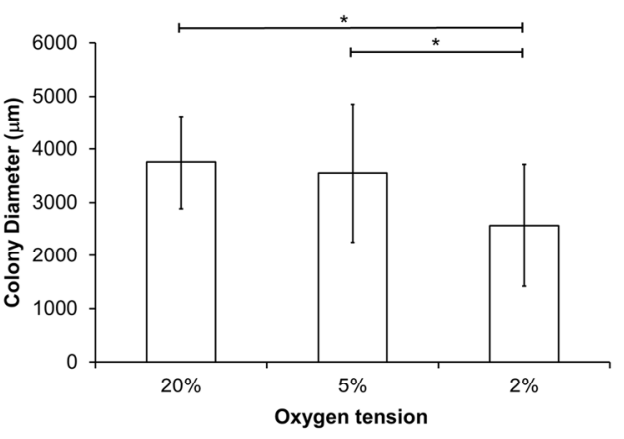

(b)

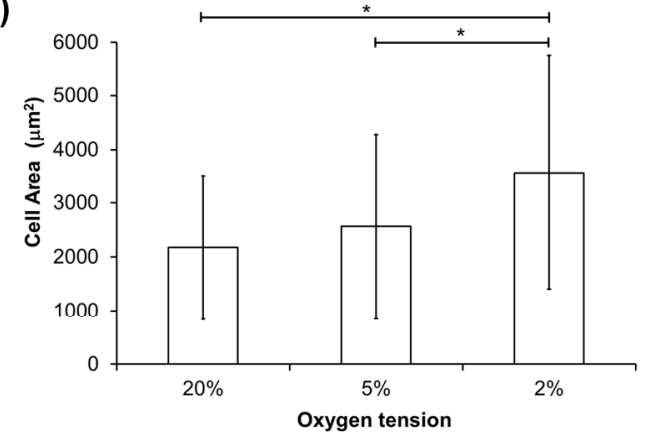


(a)
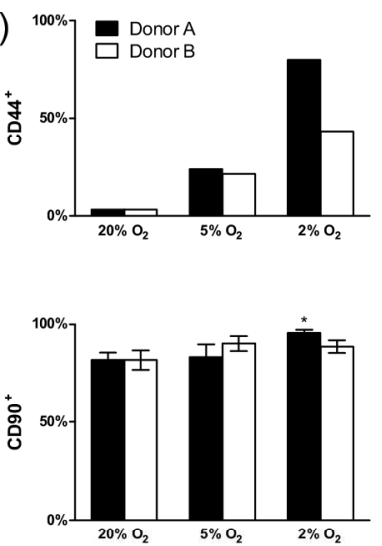

(c)
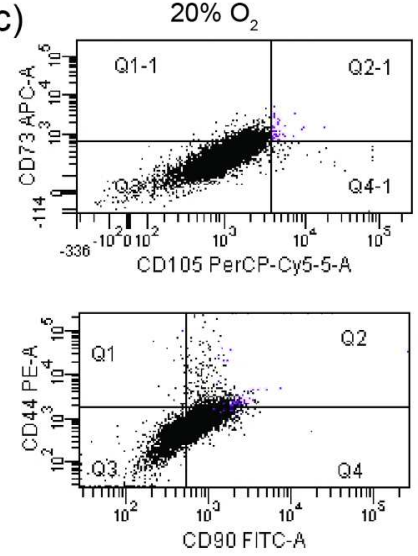
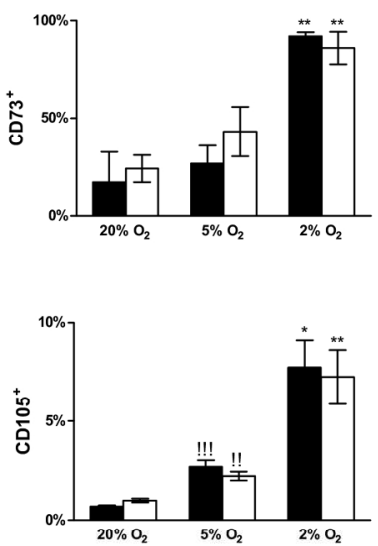

$5 \% \mathrm{O}_{2}$
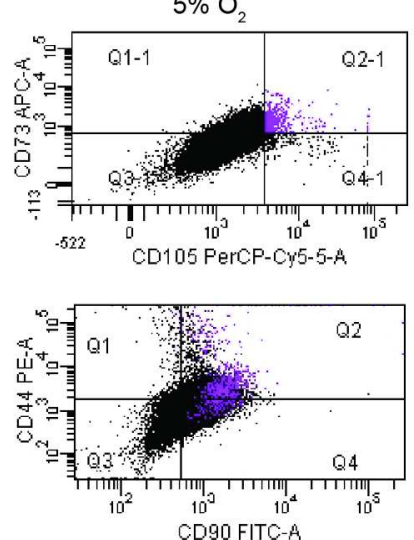

(b)
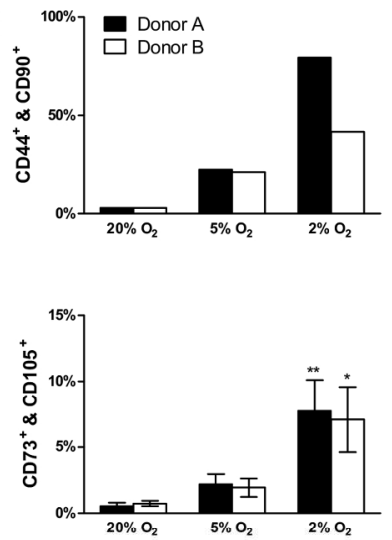

$2 \% \mathrm{O}_{2}$
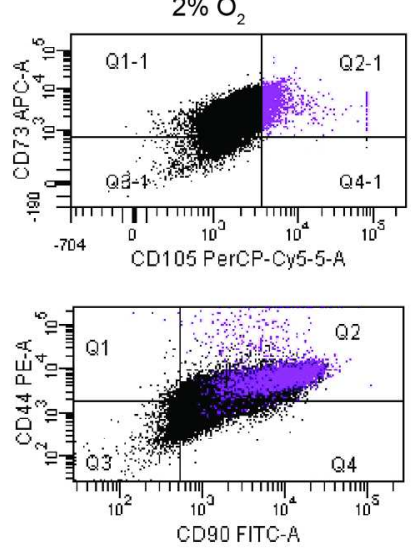

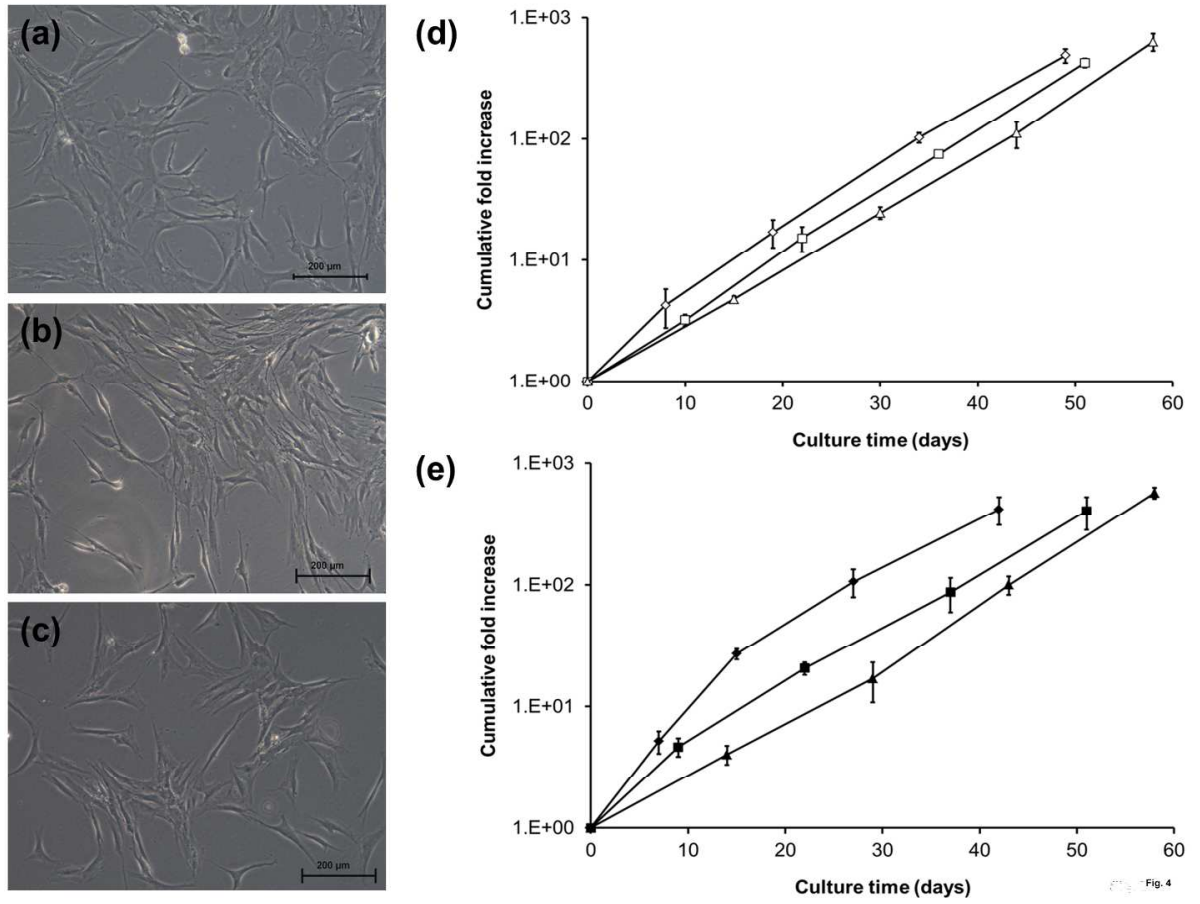


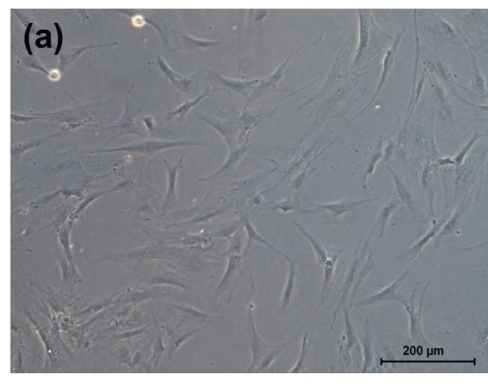

(c)
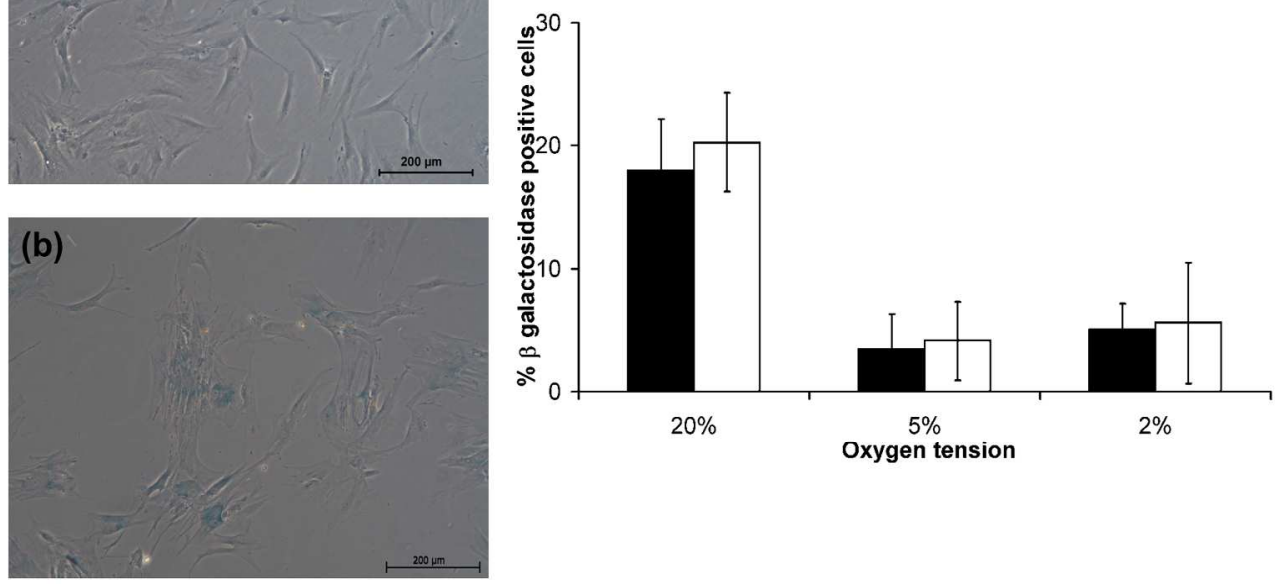

F.9. 

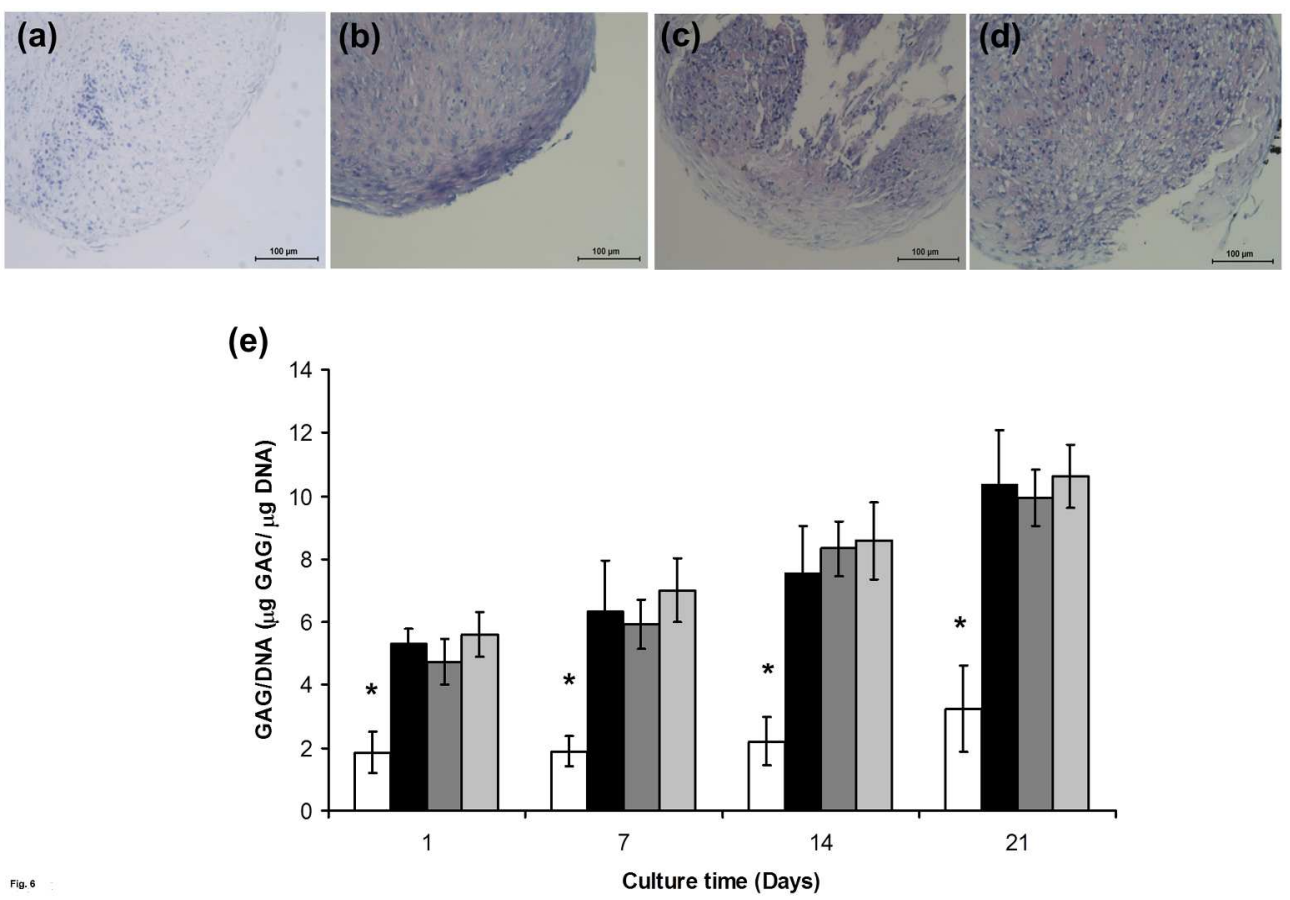
(a)
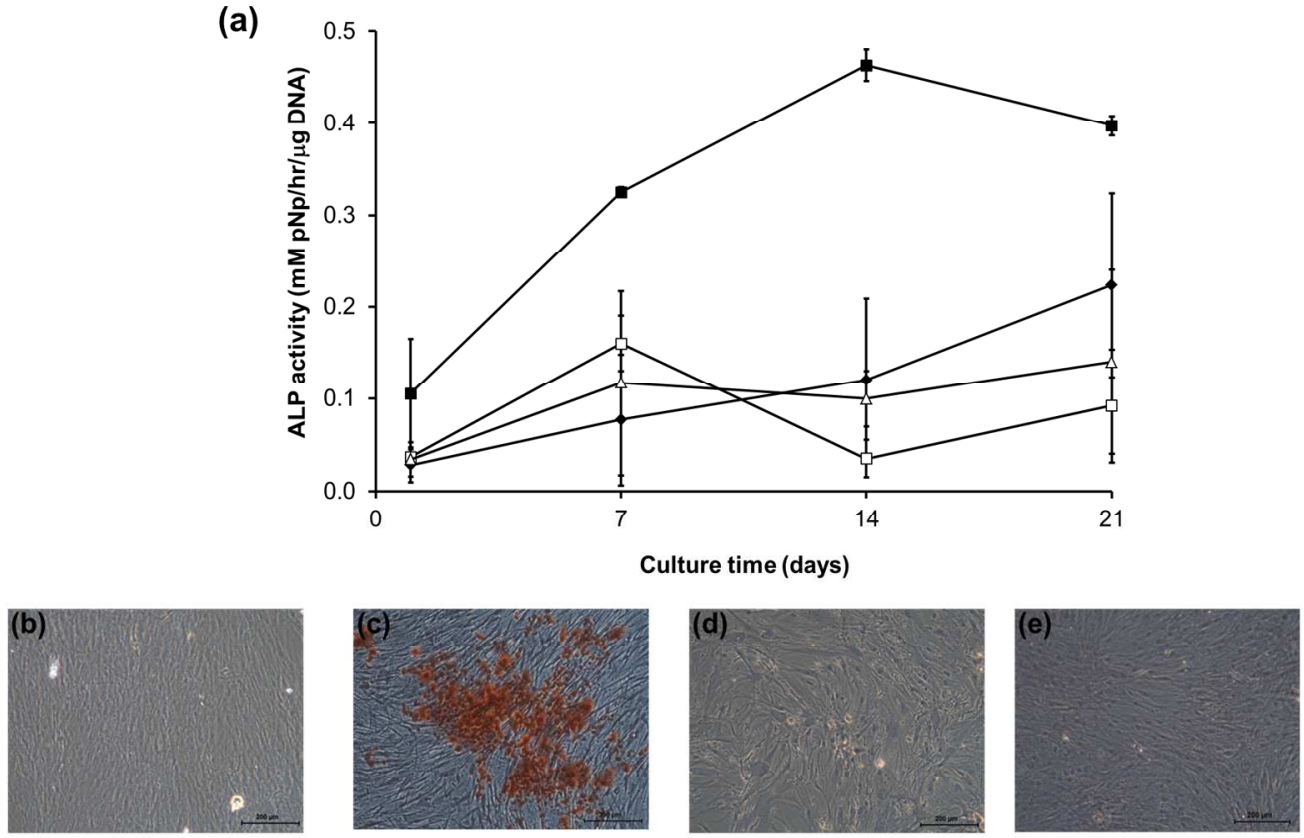

Fig. 7 

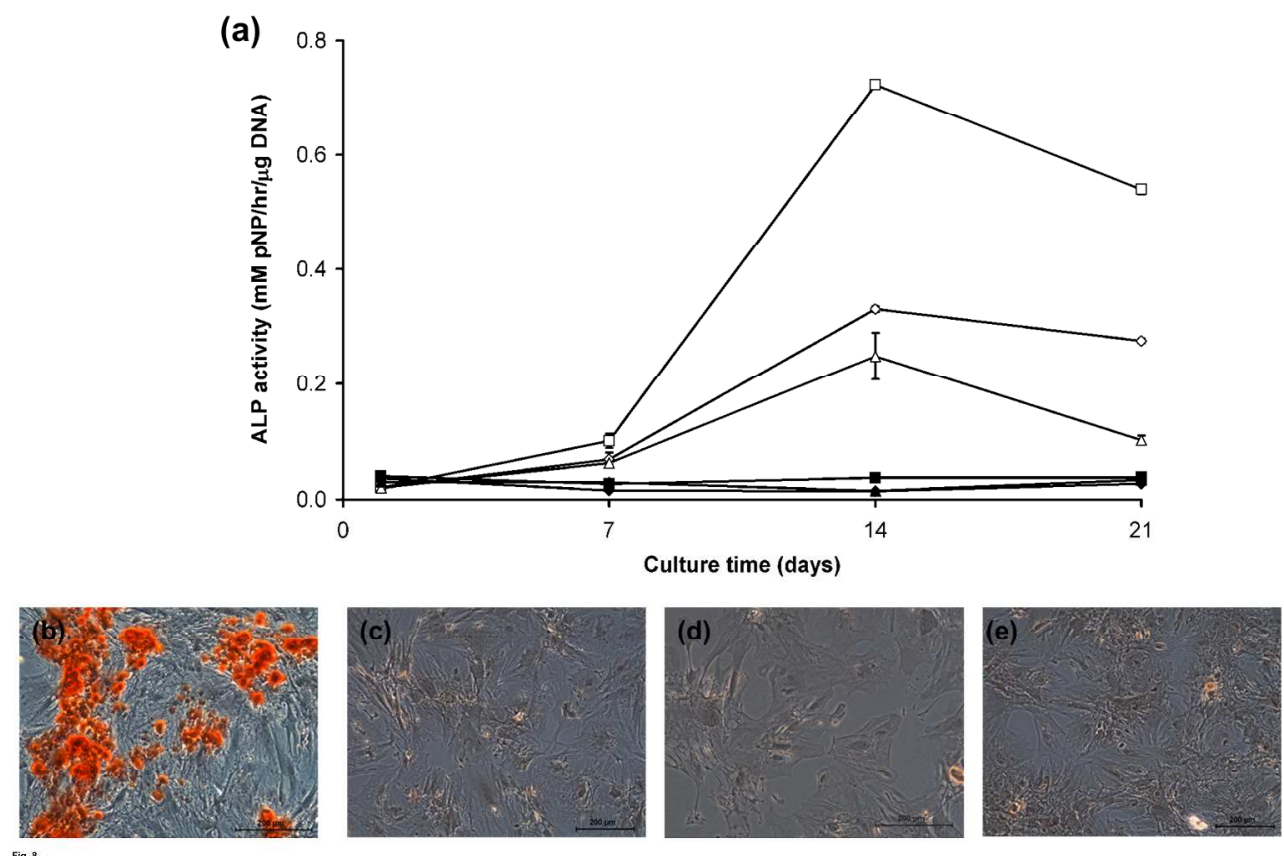

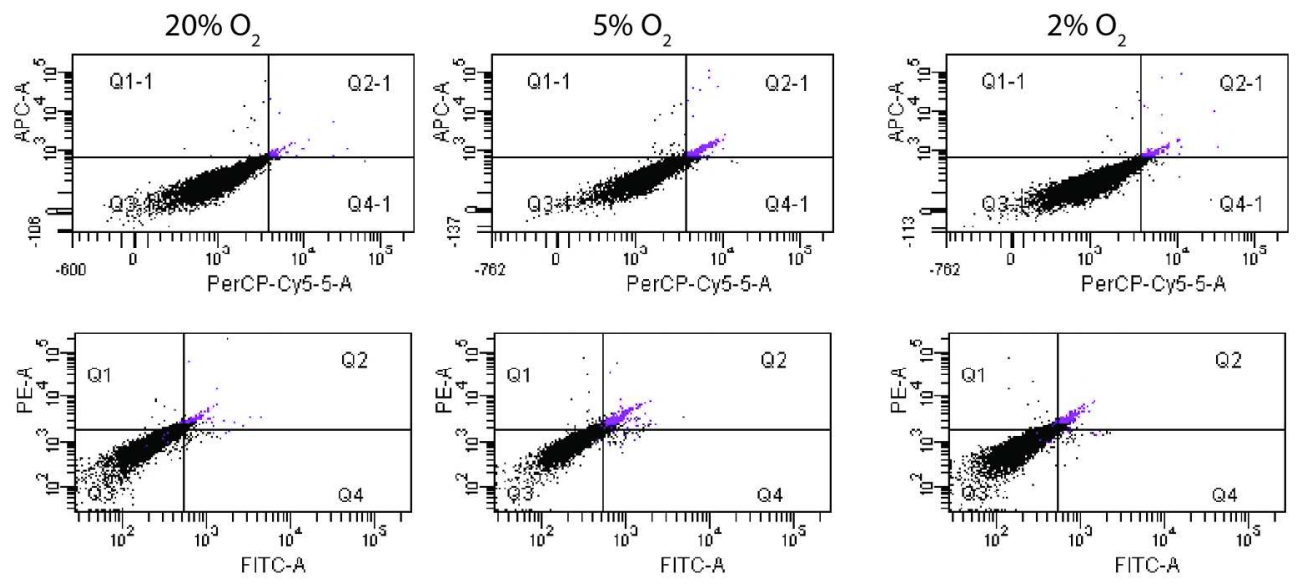

Supplemental Figure 1 


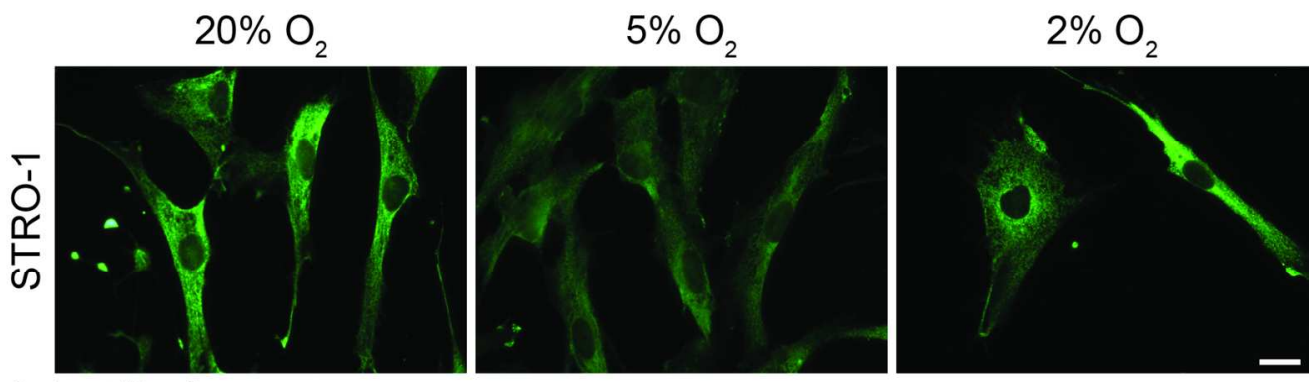

Supplemental Figure 2 
(a) Fraction of CD73+ CD105+ cell sub-population in S, G2 and M phases normalised to fraction of total analysed cells in $S, G 2$ and $M$ phases.

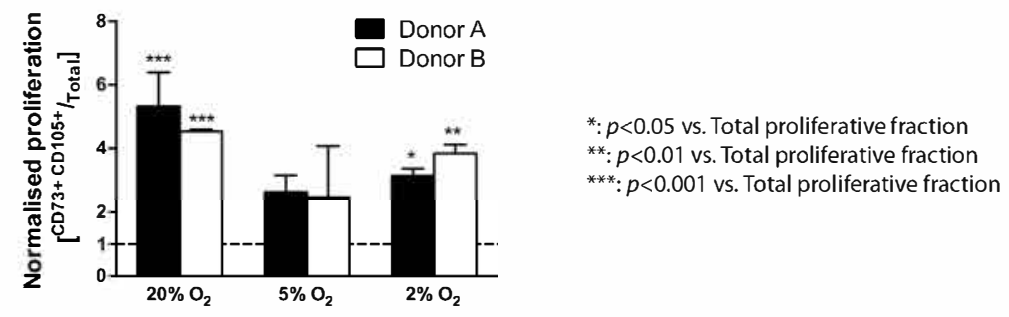

(b) Example cell cycle analysis for Donor A at 2\% oxygen with gating on specific sub-populations.
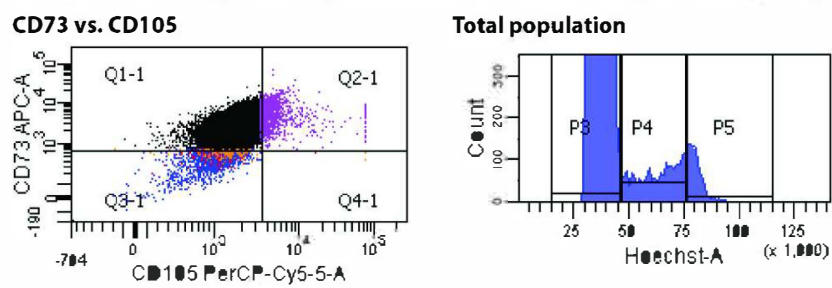

CD73+ CD105-

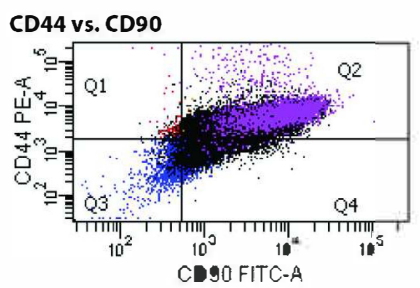

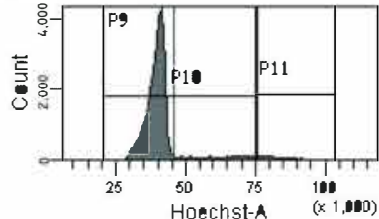

CD44+ CD90-

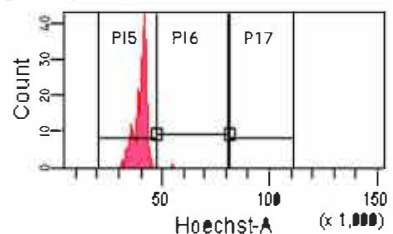

P3 G0/G1 phase All cells P6 G0/G1 phase Q2-1

P4 $S$ phase All cells P7 5 phase Q2-1

P5 G2/M phase All cells $P 8 \quad$ G2/M phase Q2-1

P9 G0/G1 phase Q1-1 P12 G0/G1 phase Q2

P10 S phase Q1-1 P13 S phase Q2

P11 G2/M phase Q1-1 P14 G2/M phase Q2

P15 G0/G1 phase Q1

P16 5 phase Q1

P17 G2/M phase Q1

CD73+ CD105+

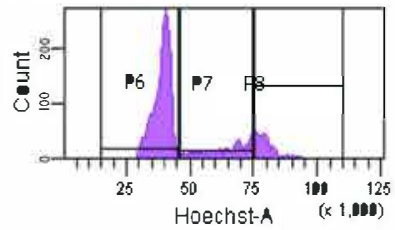

CD44+ CD90+

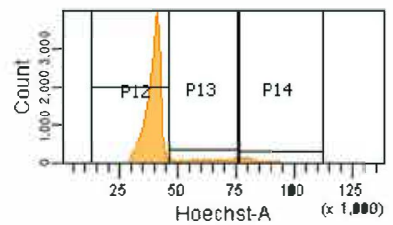

